\title{
MONOLITHIC INTEGRATION OF GAAS SAW CHEMICAL MICROSENSOR ARRAYS AND DETECTION ELECTRONICS
}

\author{
Stephen A. Casalnuovo, Vincent M. Hietala, Edwin J. Heller, Gregory C. Frye-Mason, \\ Albert G. Baca, and Joel R. Wendt \\ Sandia National Laboratories \\ Albuquerque, New Mexico 87185-1425
}

\begin{abstract}
We describe the integration of an array of surface acoustic wave delay line chemical sensors with the associated RF microelectronics such that the resulting device operates in a $D C$ in/DC out mode. The microelectronics design for on-chip RF generation and detection is presented. Both hybrid and monolithic approaches are discussed. This approach improves system performance, simplifies packaging and assembly, and significantly reduces overall system size. The array design can be readily scaled to include a large number of sensors.
\end{abstract}

\section{INTRODUCTION}

In many of chemical sensing applications, sensor size is a critical system constraint, where smaller size generally translates into lighter weight, smaller sample volumes, lower power consumption, and greater portability. Small sensors are also more readily arrayed into a system with a high degree of chemical discrimination, capable of operation in the complex chemical backgrounds found in real-world environments. However, a reduction in the size of the chemical transducer is not sufficient to reduce the overall sensor system size significantly, since system size is often determined by the control electronics, electrical interconnections, and packaging. To bring about a significant reduction in system size, these other components must be considered as well. For chemical transducers that can be produced on semiconductor substrates, the monolithic integration of the transducer with its control electronics, through the use of integrated circuit (IC) microfabrication technology, can bring about the desired size reduction. Other benefits as well often accrue with monolithic integration, including enhanced manufacturability and reliability, minimized temperature. dependence, simplified packaging and assembly, and reduced cost.

Gallium arsenide ( $\mathrm{GaAs}$ ) is an intriguing semiconductor material for the particular case of acoustic wave chemical sensors, when viewed in terms of integration. GaAs is intrinsically piezoelectric, which permits electrical generation of acoustic waves. A mature GaAs IC technology also exists, with commercial foundries able to provide application-specific integrated circuits (ASICs) from a customer's design. Coincidentally, this IC industry has driven two developments critical to integrated acoustic wave sensors: high purity, low defect density, crystalline GaAs substrates suitable for acoustic wave generation and high frequency analog ICs required to generate and detect the acoustic signals. Thus, the foundational technologies required for integrated GaAs acoustic wave chemical sensors are in place.

Acoustic wave chemical sensors operate at high frequencies, typically between $100 \mathrm{MHz}$ and $500 \mathrm{MHz}$, because chemical sensitivity generally increases with acoustic frequency [1]. This frequency range underscores the importance of monolithic integration for this particular application. In addition to the advantages listed above, a device that contains all high frequency electronic and acoustic components on a single substrate is expected to have improved performance because of a reduction in losses associated with transferring high frequency signals between discrete components. GaAs is particularly well suited for high frequency microelectronics integration, making complete dircct current (DC) -in, DC-out operation on a single chip a realizable goal.

\section{SURFACE ACOUSTIC WAVE SENSOR DESIGN}

As shown in Figure 1, surface acoustic wave (SAW) delay line sensors consist of a piezoelectric substrate and two interdigitated transducers (IDTs) formed by photolithographic patterning of a thin metal layer. Application of an alternating voltage to the input transducer generates an alternating strain field that launches a surface acoustic wave. The acoustic wave travels along the substrate surface before being converted back into an electrical signal by the output transducer. The time delay resulting from the transit of the acoustic wave between the IDTs gives rise to the name of these devices.

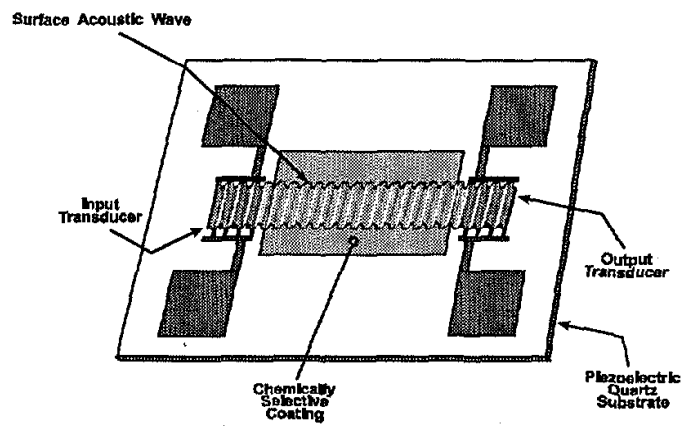

Figure 1. Schematic of a SAW delay line sensor.

The velocity and attenuation of the propagating wave are very sensitive to properties such as mass, temperature, and viscoelasticity of thin films formed on the device surface. For example, increases in surface mass loading decrease the SAW velocity. This property can be used to detect picogram mass changes [1]. By coating the acoustic path with a material that sorbs a chemical species of interest, this sensitivity can be used to develop chemical sensors [1-3]. When configured in an oscillator circuit, as shown in Figure 2, changes in the delay line acoustic velocity, $\Delta V_{R}$, which is equivalent to a phase change, $\Delta \theta$, can be observed as a frequency shift, $\Delta f$. This oscillator approach converts a very small velocity change into a measurable frequency increment, which can be determined with parts per billion resolution. In the limit of small perturbation, the response to these surface changes can be expressed as:

$$
\frac{\Delta f}{f_{o}}=\frac{\Delta \theta}{\theta_{o}}=\frac{\Delta V_{R}}{V_{R o}}=-k_{m} \frac{\Delta m}{m_{o}}-k_{T} \frac{\Delta T}{T_{o}},
$$

in which $m$ is the surface mass, $T$ is the temperature, $k_{m}$ is the mass sensitivity and $k_{T}$ is the temperature sensitivity. 


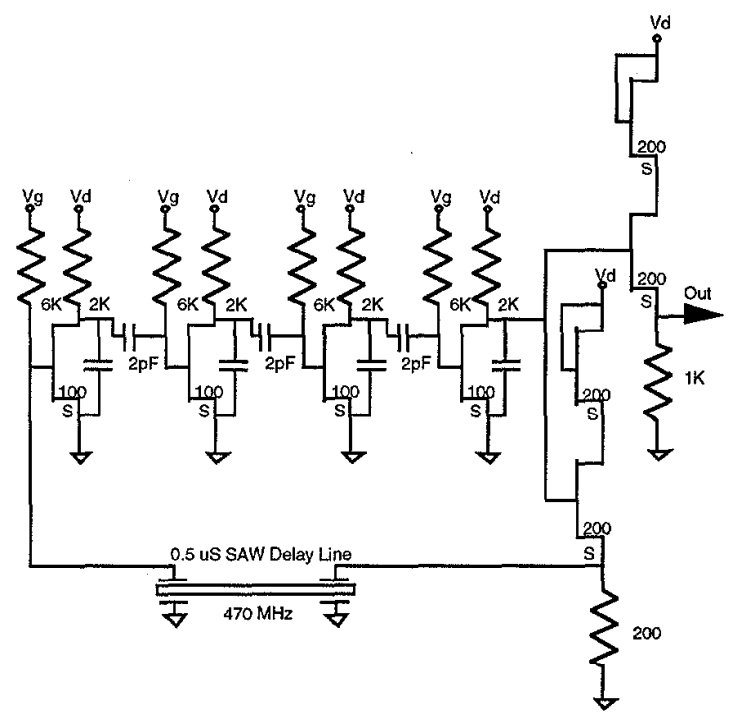

Figure 2. Schematic of a $470 \mathrm{MHz}$ SAW oscillator circuit.

GaAs SAW delay lines are fabricated on (100) semiinsulating substrates with the delay lines oriented in the [011] direction. These substrates are standard for microelectronics applications and the [011] direction maximizes the coupling between electromagnetic and acoustic waves. These delay lines operate between $100 \mathrm{MHz}$ and $500 \mathrm{MHz}$, where the pitch of the IDT finger pairs determines the frequency of the device. Typically, each IDT contains 50 finger pairs. The input and output IDTs are separated by 190 acoustic wavelengths and are 30 wavelengths wide. IDTs consist of a $500 \AA$ to $1000 \AA$ thick gold layer (depending on device frequency) on top of a $200 \AA$ thick titanium adhesion layer, deposited by thermal evaporation.

\section{SAW SENSOR ARRAY}

Because of the limited selectivity of the sorbing coatings applied to SAW chemical sensors, a single SAW sensor is insufficient to identify analytes in a realistic background of interferants. In practice, an array of sensors, each with a different selective coating, is used to make reliable chemical identification. An array of sensors also allows redundancy and error-checking, important advantages in the overall system. Multiple sensors are also commonly used to compensate for undesired sensor responses. In a classic dual sensor system, two sensors are maintained in identical environments except one is introduced to the desired stimulus. The resulting responses (frequencies) can then be subtracted to allow for removal of the undesired background responses due to temperature or other surface changes.

Figurc 3 shows a block diagram of a novel dual-sensor approach that allows for integration and can be scaled to larger array size. In this system, phase is compared (subtracted) rather than frequency. One SAW device ( $\mathrm{Ch} R$ ) is used as a reference channel and is assumed isolated from the surface stimulus of interest. The reference oscillator drives a second acoustic channel (termed test channel, $\mathrm{Ch} \mathrm{T}$ ) on the same substrate. Note the unique use of the acoustic wave propagating in either direction from the center IDT to provided the necessary power split of the reference and test channels. Multiplying the test signal with the reference signal and observing the DC component of the result performs the phase difference. A parasitic second harmonic is present at the phase output and is filtered off. It should be noted that this approach has converted the velocity change into a voltage change. This allows for easy measurement with low-cost electronics, but also undoubtedly loses resolution compared to a time measurement.

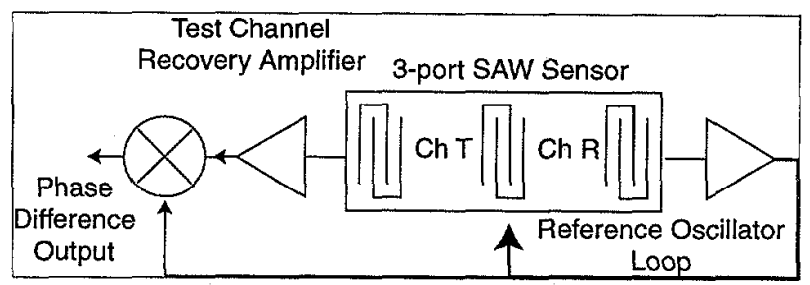

Figure 3. Block diagram of a dual-channel (3-port) SAW sensor system.

This design compensates for changes in the sensor temperature. Since the two acoustic paths are on the same substrate, they can be assumed to be at exactly the same temperature. Therefore, referring back to Equation (1) and assuming that only the test channel only experiences the mass uptake, the temperature effects will exactly cancel and only the mass uptake of the test channel will be present at the output. This compensation is almost perfect except for the slight change in mass sensitivity as the reference channel center frequency changes over temperature. Additionally, this approach is tolerant to "modehopping" (changes in the longitudinal mode number) due to the subtractive nature of the technique. In practice, the necessary application of the chemically selective thin film to the test channel will change the temperature coefficient of the test channel and compromise the complete elimination of the temperature terms in the subtraction. This unfortunate effect will be an issue for any measurement approach and substrate material.

\section{SAW ARRAY ELECTRONICS DESIGN}

The dual-channel temperature compensated SAW sensor approach is readily extended to large arrays of acoustic sensors. A schematic of a four-element SAW delay line array configuration is shown in Figure 4. A large bi-directional central transmitting IDT spans each of four smaller receiving transducers. Appropriate chemically sorbent coatings are applied to three of the regions between the transmitting IDT and the receiving IDTs, with the fourth left uncoated to provide a reference phase for the array. As in the dual sensor system, an oscillator circuit drives the transmitter, launching an acoustic wave to the receivers. Phase comparator circuitry measures the relative phase of each of the receivers with respect to the reference and provides a DC output voltage proportional to the relative phase. The block diagram of a four-channel system, including electronic circuitry, is shown in Figure 5. Extension to n-channels simply requires splitting the reference signal $2 n-1$ ways ( $n$ inputs for each acoustic channel and mixer drive for $n-1$ channels).

The electronics diagramed in Figure 5 incorporate several novel design features. All signals represented by single lines in this figure are implemented differentially in the actual design. The amplifier blocks shown as triangles are specially designed limiting differential amplifiers providing small phase errors at large overdrive conditions. The amplifiers also provide a log output signal, which allows for an accurate power measurement of the signals from each SAW output port. This permits measurement of the insertion loss of each SAW channel. The mixers are implemented as Gilbert cell mixers, which homodyne the test signal to DC. The input signals to the mixers are hard limited (square waves) by the 
amplifiers so in effect the mixer operates digitally, performing the exclusive-or function. This provides linear phase detection and provides two-quadrant phase detection as shown in Figure 6. This detection response provides a unique output if the two signals' phase difference remains within the appropriate two quadrants of phase space ( 1 and 2 or 3 and 4 ).

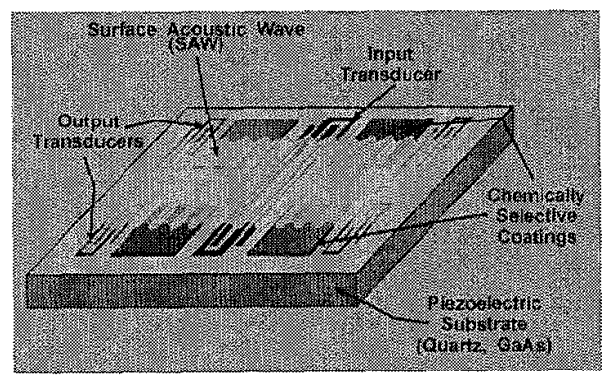

Figure 4. Schematic of an array of four surface acoustic wave devices on a single substrate.

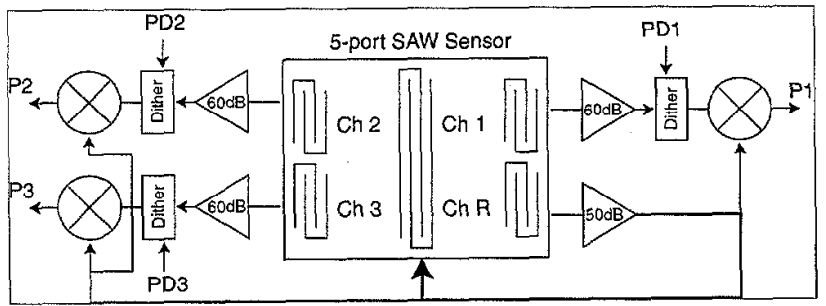

Figure 5. Block diagram of a four-channel (5-port) SA W sensor system.

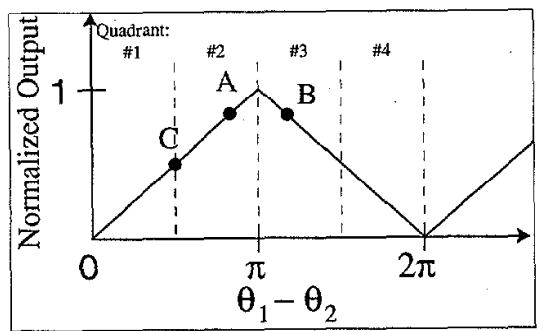

Figure 6. Diagram of the normalized DC component from the Gilbert cell mixer.

To achieve the desired full four-quadrant phase detector, the phase "dither" circuits as shown in the block diagram were added. The phase dither circuits allow for a given test channel to be selectively advanced or retarded in phase by a fixed predetermined amount. Inputs arc provided to cycle through three phase states to determine the phase uniquely over the complete $2 \pi$ phase space. Operation of the dither sequence can be illustrated by an example shown by the three labeled points in Figure 6 . Assume the actual phase is at the point A. From a single DC output measurement, one cannot determine if the actual phase is in quadrant $\# 2$ or quadrant $\# 3$ (normalized output is the same at points $A$ and $B$ ). Using the phasc dither, the response curve can be "shifted" to determine the "slope" of the output and hence the correct quadrant. Assuming that the dither shifts the output from point $A$ to point $C$, the negative slope indicates the actual point is in quadrant \#2. Three dither states are provided to handle the event where the measured and dithered points straddle a quadrant boundary (as with points $A$ and $B$ in Figure 6). In general all three dither states must be considered to guarantee unique phase detection over all phase space.

\section{HYBRID FOUR-ELEMENT SAW ARRAY}

An array of SAW sensors and the associated microelectronics was first produced using a hybrid packaging approach rather than through monolithic integration [4]. Although it requires more assembly than the monolithic approach, the hybrid device prototypes can be produced more rapidly and can be used to test the sensor design and circuit functionality. It also represents a significant size reduction compared to conventional SAW sensor systems.

The SAW array is fabricated on a ST-quartz substrate. In this case, quartz was selected because the requirement for monolithic integration was removed and quartz has slightly better aconstic performance than GaAs. The oscillator amplifier and phase comparator circuitry are custom GaAs ASICs attached directly to the quartz substrate. Metal paths patterned directly onto the quartz die provide circuit interconnection. Wire bonding is used to connect the ICs to the metal paths on the quartz. Although not monolithically integrated, this device incorporates all high frequency components on the quartz substrate so that the packaged part operates in the desired DC in/DC out mode. A photograph of the multi-chip SAW array configuration is shown in Figure 7. The hybrid device requires $90 \mathrm{~mA}$ at $2.5 \mathrm{~V}$ to operate the GaAs ASICs, which can readily be provided by batteries. The SAW oscillator operates at $510 \mathrm{MHz}$ and the phase comparators have a sensitivity of $1 \mathrm{~V}$ per 180 degrees of phase.

These devices have been tested as chemical sensors. The response of the SAW array to dimethyl methyl phosphonate (DMMP) is shown in Figure 15. The varied response of the 3 different coatings indicates their relative sensitivity to DMMP and demonstrates how the array can be used to discriminate among a number of analytes and interferants.

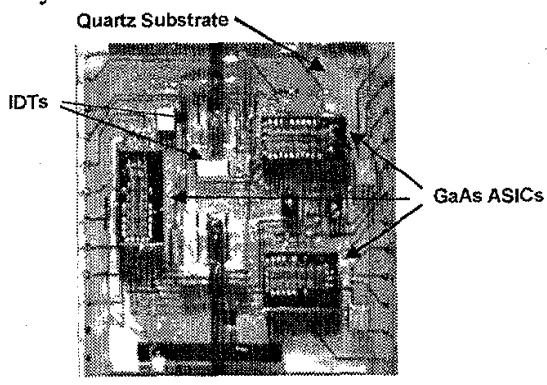

Figure 7. Hybrid version of SAW microsensor array. The STquartz die size is $6.9 \mathrm{~mm}$ by $8.6 \mathrm{~mm}$. Each of the GaAs ICs is approximately $1 \mathrm{~mm} \times 2 \mathrm{~mm}$. The device operates in a $D C$ in/DC out mode.

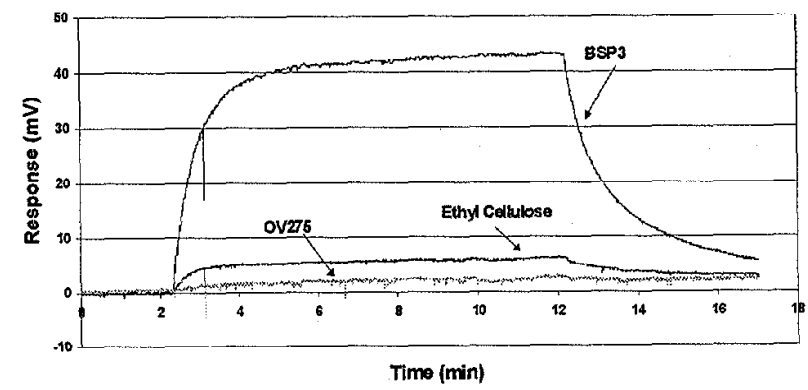

Figure 8. Hybrid SAW array response to 15 parts per million of DMMP. The three microsensor coatings were BSP3 hydrogenbond acid, ethyl cellulose, and oV-275 (a cyano-modified polysiloxane). 


\section{MONOLITHIC SAW SENSOR ARRAY}

We have completed fabrication of the first fully monolithic version of the SAW sensor array described above. This device is based on a GaAs SAW array of the same configuration shown in Figures 4 and 5 and uses the same GaAs microelectronic circuitry as the die shown in Figure 7, but puts all these components onto a single GaAs substrate. Figure 9 (a) shows one of the four-element arrays with the four delay lines arranged diagonally across the center of the die and the amplifier and phase comparator circuitry placed in the comers. The die measures $4.6 \mathrm{~mm}$ by $4.6 \mathrm{~mm}$. The microelectronics on the monolithic devices are fabricated at a commercial GaAs IC foundry. The partially processed wafers are transferred to our laboratory for IDT fabrication. Prior to IDT fabrication, a plasma etch is used to remove several microns of dielectric material to expose the GaAs surface. The IDTs are then patterned on the GaAs surface using a metal lift-off process. Figure 9 (b) shows the frequency spectrum of the delay line oscillator portion of the device, which operates at approximately $692.5 \mathrm{MHz}$ and draws $28.5 \mathrm{~mA}$ at $3 \mathrm{~V}_{\mathrm{dc}}$. Oscillator function demonstrates that the post-processing of the IDTs is compatible with the microelectronics fabrication process. The temperature dependence of the phase comparator output has been tested and this is insensitive to temperature, as expected. These devices are now bcing packaged for further electronic and chemical testing.

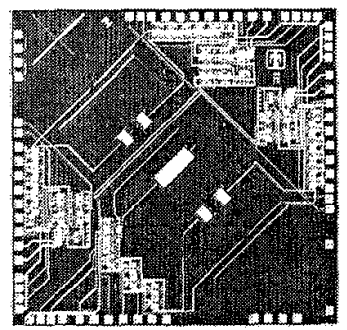

(a)

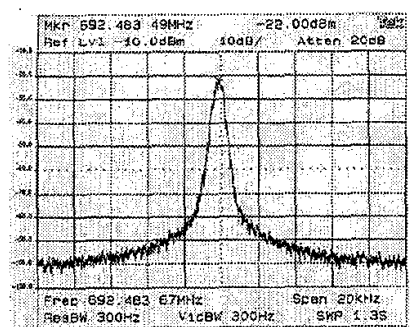

(b)
Figure 9. Monolithic GaAs SAW sensor array. (a) Photograph of die showing IDTs in the center and microelectronics in the corners. Die size is $4.6 \mathrm{~mm} \times 4.6 \mathrm{~mm}$. (b) Frequency spectrum of integrated oscillator, demonstrating successful delay line integration.

\section{SUMMARY}

We have described the development of microfabricated SAW chemical sensor arrays, culminating in a fully monolithic device that integrates all high frequency components onto a single GaAs substrate. These arrays operate in a DC in/DC out mode that simplifies assembly and improves performance. Hybrid arrays show good sensitivity to chemical analytes. Monolithic arrays have shown electronic functionality and are undergoing chemical tests.

\section{ACKNOWLEDGEMENTS}

Sandia is a multiprogram laboratory operated by the Sandia Corporation, a Lockheed Martin company, for the United States Department of energy under Contract DE-AC04-94AL 85000. The authors wish to thank John Reno and John Klem for providing the GaAs/AlGaAs epitaxial materials; Tim Drummond for providing the ion implantation for the monolithic SAW delay line oscillators;
Richard Kottenstette and Pat Lewis for testing the chemical sensors; and Jay Grate at Pacific Northwest National Laboratory for providing some of the chemically selective coatings.

\section{REFERENCES}

1. D. S. Ballantine, Jr., R. M. White, S. J. Martin, A. J. Ricco, E. T. Zellers, G. C. Frye, and H. Wohltjen, Acoustic Wave Sensors Theory, Design, and Physico-Chemical Applications, Academic Press, New York, (1997).

2. G. C. Frye, D. S. Blair, T. W. Schneider, C. D. Mowry, C. C. Colburn, and R. P. Donovan, "Development and Evaluation of OnLine Detection Techniques for Polar Organics in Ultrapure Water", Journal of the Institute Of Environmental Sciences, 39, 30 (1996).

3. J. W. Grate, S. L. Rosepehrsson, D. L. Venezky, M. Klusty, H. Wohltjen, "Smart Sensor System for Trace Organophosphorous and Organosulfur Vapor Detection Employing a TemperatureControlled Array of Surface-Acoustic-Wave Sensors, Automated Sample Preconcentration, and Pattern-Recognition", Analytical Chemistry, 65, 1868 (1993).

4. E. J. Heller, V. M. Hietala, R. J. Kottenstette, R. P. Manginell, C. M. Matzke, P. R. Lewis, S. A. Casalnuovo, and G. C. FryeMason, "An Integrated Surface Acoustic Wave-Based Chemical Microsensor Array for Gas-Phase Chemical Analysis Microsystems," Chemical Sensors IV, Electrochemical Society, 9923, (1999), pp. $138-142$. 